\title{
A Pilot Investigation of the Graduated Recovery Intervention Program (GRIP) for First Episode Psychosis
}

\author{
David L. Penn, Ph.D ${ }^{1,2,3}$, Sarah R. Uzenoff, M.A. ${ }^{1}$, Diana Perkins, M.D ${ }^{2,3}$, Kim T. Mueser, \\ Ph.D. ${ }^{4}$, Robert Hamer, Ph.D. ${ }^{2}$, Evan Waldheter, Ph.D ${ }^{1}$, Sylvia Saade, Ph.D ${ }^{2,3}$, and Liz \\ Cook, M.A. ${ }^{2}$ \\ ${ }^{1}$ University of North Carolina-Chapel Hill, Department of Psychology \\ ${ }^{2}$ University of North Carolina-Chapel Hill, Department of Psychiatry \\ ${ }^{3}$ University of North Carolina-Chapel Hill, Outreach and Support Intervention Services Program \\ ${ }^{4}$ Dartmouth Medical School
}

\begin{abstract}
The Graduated Recovery Intervention Program (GRIP) is a new individual cognitive-behavioral therapy program designed to facilitate functional recovery in people who have experienced an initial episode of psychosis. The purpose of this study was to evaluate the feasibility and tolerability of the GRIP intervention, and to compare the effectiveness of GRIP versus treatment as usual (TAU) for improving specific clinical and psychosocial outcomes. Forty-six individuals with first episode psychosis were randomized to GRIP + TAU or TAU alone. Primary outcomes focused on social and role functioning, and quality of life. Secondary outcomes included psychotic symptoms, depression, substance use, social support, attitudes toward medications, well-being, and hospitalizations. The results indicate that GRIP was well-tolerated, as evidenced by good attendance and low drop-out rates, and well-received (based on positive feedback from participants). Although the majority of mixed-model analyses were not statistically significant, examination of within-group changes and effect sizes suggest an advantage for GRIP over TAU in improving functional outcomes. These advantages and the fact that the GRIP intervention demonstrated feasibility and tolerability suggest that this intervention is worthy of further investigation.
\end{abstract}

\section{Keywords}

psychosocial treatment; early psychosis; functional recovery

\section{Introduction}

There is emerging evidence that sustained and targeted intervention early in the course of psychotic disorders is essential in order to facilitate recovery from a first episode, reduce the risk of relapse and re-hospitalization, and minimize psychosocial deterioration (Birchwood, Todd, \& Jackson, 1998; Malla, Norman, \& Joober, 2005). One approach to early

\footnotetext{
(C) 2010 Elsevier B.V. All rights reserved.

This is a PDF file of an unedited manuscript that has been accepted for publication. As a service to our customers we are providing this early version of the manuscript. The manuscript will undergo copyediting, typesetting, and review of the resulting proof before it is published in its final citable form. Please note that during the production process errors may be discovered which could affect the content, and all legal disclaimers that apply to the journal pertain.
} 
intervention is via individual therapy (J. Addington \& Gleeson, 2005). In general, individual therapy has been shown to reduce suicidal ideation and hopelessness (Power et al., 2003), substance use (Kavanagh et al., 2004), positive symptoms (Uzenoff, Perkins, Hamer, Wiesen, \& Penn, 2008), relapse rates (Gleeson et al., 2009), and increase activity levels (Fowler et al., 2009), although there are exceptions (J Edwards et al., 2006; Nicholas Tarrier, Haddock, Lewis, Drake, \& Gregg, 2006). Such individual therapy approaches may be more effective later rather than earlier in the course of recovery (i.e., when clients are more stable) (C. Jackson et al., 2009; Lewis et al., 2002; Nick Tarrier et al., 2004), and it remains unclear whether group treatment confers greater benefits than individual treatments (Lecomte et al., 2008; Saksa, Cohen, Srihari, \& Woods, 2009). Overall, individual therapy appears to have promise as a front line intervention for first episode psychosis (Haddock \& Lewis, 2005; Harvey, Lepage, \& Malla, 2007; Penn, Waldheter, Perkins, Mueser, \& Lieberman, 2005).

Despite the encouraging findings for individual therapy for first episode psychosis, there have been no controlled evaluations of this treatment approach in the United States. In addition, the targets of extant individual therapy approaches for an early psychosis population have often been narrowly defined, emphasizing only one or two areas of concern for individuals recovering from a first episode, such as symptoms (Lewis et al., 2002) or substance use (J. Edwards, Hinton, Elkins, \& Anthanasopoulos, 2003), and not functional recovery (for an exception, see (Fowler et al., 2009). Thus, the impact of individual therapy on first episode psychosis seems to be more domain-specific than broad-based.

To address these unmet needs, we developed the Graduated Recovery Intervention Program (GRIP). GRIP is a comprehensive, flexible, modular-based cognitive-behavioral therapy program that aims to enhance illness management and facilitate functional recovery (i.e. social skill, role and community functioning) following a first episode of psychosis. The GRIP program places an emphasis on personal goal pursuit to foster optimism and selfesteem, targets malleable factors that may enhance recovery such as residual symptoms and substance use, and enlists external social support to maximize therapeutic gains and engagement.

Following a thorough treatment development period (i.e., writing of the manual; obtaining feedback from clients, clinicians, and researchers; revising the manual) (Waldheter et al., 2008), we conducted an open-trial of GRIP with 10 individuals with first episode psychosis. Qualitative feedback and ratings indicated that GRIP was well-received by clients and clinicians, and it was associated with reduced positive symptoms at post-treatment (Waldheter et al., 2008). Reductions in symptoms and improvements in social functioning and goal attainment were especially pronounced for treatment completers (i.e. clients who received at least 12 sessions), underscoring the importance of treatment engagement. These findings led to manual revisions for developing better strategies for retention (e.g. by offering GRIP on a more flexible basis) and adding content on managing grief and loss following an initial psychotic episode.

Based on the promising findings of the open trial, we designed a preliminary, small-scale randomized controlled trial (RCT), comparing GRIP + Treatment as Usual (TAU) versus TAU alone. TAU was provided in the context of a specialty first episode program in Chapel Hill, NC, Outreach and Support Intervention Services (OASIS). The primary purpose of this trial was to examine the feasibility and tolerability of GRIP. The secondary purpose was to obtain specific treatment parameters (e.g. effect sizes and variances, with confidence intervals) that may inform the implementation of a larger, more definitive RCT (although extrapolating effect sizes from pilot to large scale randomized controlled trials need to be done cautiously) (Kraemer, Mintz, Noda, Tinklenberg, \& Yesavage, 2006). This approach is 
consistent with pilot interventions early in development (Mueser \& Drake, 2005). We expected that GRIP would be well-tolerated, as measured by good attendance and low dropout rates, and received (based on feedback from clients), and that it would be associated with a greater reduction in symptoms and substance use, improvement in functioning, and lower hospitalization rates than TAU alone.

\section{Methods}

\subsection{Participants}

The participants in this RCT comparing GRIP + TAU to TAU alone were recruited from the UNC OASIS program (described below), with the exception of two individuals who were recruited from another UNC outpatient program (the Schizophrenia Treatment and Evaluation Program; STEP). Study participants were individuals with early psychosis meeting the following inclusion criteria: a) 18 years of age or older; b) current or past diagnosis of schizophrenia, schizophreniform disorder, or schizoaffective disorder according to the SCID-P, and; c) within the first three years of treatment for psychosis. Exclusion criteria included: a) current dependence on alcohol or drugs; b) history of mental retardation (i.e. IQ<70), and c) history of significant neurological disorder.

\subsection{Treatment Groups}

2.2.1. GRIP-The development of GRIP has been described in a recent report (Waldheter et al., 2008). GRIP is provided on an individual, weekly basis for up to 36 sessions, and comprises four phases: (1) engagement and wellness management; (2) substance use; (3) persistent symptoms; and (4) functional recovery. The first 12 sessions of GRIP (i.e., psychoeducation, processing the illness, goal setting, illness management, relapse prevention, and psychoeducation about substances) are relatively standardized with respect to treatment foci. During the first phase, the client is asked to identify an "indigenous supporter" (a friend, relative, or support person who they see on a regular basis) who can assist the client with homework and goal pursuit (Tauber, Wallace, \& Lecomte, 2000). The clinician is instructed to communicate periodically (at least monthly) with indigenous supporters to track client progress and troubleshoot potential problems and issues.

After 12 sessions have been completed, progress is evaluated via reviewing goal attainment and the client and therapist collaboratively determine whether additional treatment is necessary (i.e., in the case of incomplete goals, identification of new goals, or the persistence or emergence of problem areas or symptoms) and if so, which module(s) are pursued. The remaining sessions are flexible and individually tailored to the needs of the client. Therapists are encouraged to follow the prescribed order of treatment phases; however, pressing client concerns are always prioritized, regardless of phase. Based on an individual assessment, clients can receive assistance with current substance use problems (module two), persistent positive and/or negative symptoms (module three), and functional impairments (module four). Intervention techniques include psychoeducation, cognitive behavioral therapy (CBT) (i.e., for addressing residual symptoms), motivational interviewing (i.e. for substance use, if necessary), and social skills training (to enhance social functioning).

2.2.2 TAU-The OASIS program is a comprehensive, multi-element clinic for the treatment of early psychosis. The clinic is operated by the UNC Health Care System, a notfor-profit integrated healthcare system, owned by the State of North Carolina and based in Chapel Hill, NC. Consistent with the program's emphasis on community outreach and accessibility, the clinic is located separate from the primary hospital complex in an easily accessible office suite. 
OASIS serves adolescents and young adults aged 16-36 who have been treated for psychosis (i.e. have been taking antipsychotic medications) for no more than three years, as well as previously unmedicated individuals who have been ill for up to 5 years. Referrals to OASIS come from several sources, primary among which are the inpatient and emergency treatment services of the UNC Hospital System. Additional referral sources include the UNC counseling center, UNC STEP, family members, and community mental health providers.

Every client is assigned a primary clinician (a licensed or licensed eligible social worker) who provides clinical services and case management based on individual need. Interventions range from engagement techniques (i.e. making phone calls to engage reluctant or withdrawn clients) to various aspects of case management, to providing individual supportive and cognitive-behavioral therapy. Services are frequently provided in the community or at the client's home in addition to in the office. Each client also receives psychiatric assessment and medication management from a psychiatrist. In addition, family members are encouraged to attend either single or multi-family treatment. OASIS offers time-limited groups lasting for 8-12 weeks depending on client interest and need. Topics include psychoeducation, healthy lifestyle habits, stress management, substance abuse and social skills training. Finally, a certified substance abuse counselor provides substance abuse assessment, individual therapy and leads a group following the integrated dual diagnosis modality of treatment. Clients' utilization of these services depends on their need and interest, and treatment team recommendations.

2.2.3 Therapists and Fidelity-Three therapists provided GRIP: two social workers (each seeing 10 clients) and one clinical psychologist (the PI, who provided treatment to three GRIP clients). The social workers were trained over approximately a one month period via didactic presentations, required readings, role plays, and listening to therapy tapes from the open trial. The PI (who served as clinical supervisor) further evaluated competence via listening to every therapy session and providing detailed feedback/comments to therapists during weekly supervision meetings. Audiotaped therapy sessions were later rated by either the PI or Co-I (Kim T. Mueser, Ph.D.) for fidelity to the GRIP manual (NOTE: the fidelity manual was developed following the open trial). Twenty two percent of the total sessions were rated for treatment fidelity. Overall, the therapists received overall mean quality ratings from 3.78 to 4.21 (range $=1-5$ ), indicating good to very good ratings, which reflect how well the rater believes the therapist implemented the prescribed techniques, and considering issues of therapist demeanor, style and skill.

\subsection{Measures}

Participants were assessed at baseline, post-treatment, and 3-month follow-up on measures of functioning (addressed throughout GRIP, but predominantly in Module \#4), psychiatric symptoms (addressed in Modules \#1 and \#3), substance use (addressed in Modules \#1 and \#2), mood (which we expect to improve as illness management and goal achievement occurs), social support (addressed throughout GRIP, but predominantly in Module \#4), attitudes toward medications (addressed in Module \#1), well-being (which is a more distal outcome that we expect to improve after goal achievement and functional recovery occur), and hospitalizations.

Below, the battery is summarized in terms of screening measures (prior to baseline), and primary and secondary outcomes. 


\subsection{Screening Measures}

Diagnosis was verified by the Structured Clinical Interview for DSM-IV (SCID-P) and a chart review.

IQ was estimated using the Wechsler Abbreviated Scales for Intelligence (WASI) (The Psychological Corporation, 1999), which is comprised of Matrix Reasoning, Vocabulary, Similarities, and the Block Design subtests.

\subsection{Primary Outcomes}

The primary outcomes for this study focused on functional outcomes and included quality of life, community functioning, and social skill.

Quality of life was measured using the Quality of Life Scale (QLS) (Heinrichs, Hanlon, \& Carpenter, 1984). This assessment is a semi-structured interview comprising 21 items rated on a scale from 0 to 6. Lower scores reflect greater impairments in functioning. The QLS generates four subscales (Interpersonal Relations, Instrumental Role, Intrapsychic Foundations, and Common Objects and Activities). In the current study, we did not include the last two subscales, but rather focused on the Interpersonal Relations subscale (8 items) and Instrumental Role subscale (4 items), due to the emphasis of the treatment study on functional recovery.

Community functioning was measured with the Role Functioning Scale (RFS) (Goodman, Sewell, Cooley, \& Leavitt, 1993) and the Multnomah Community Ability Scale (MCAS) (Dickerson, Origoni, Pater, Friedman, \& Kordonski, 2003). Ratings on the RFS were based on information obtained from interviews conducted with the participant's significant other and were based on the participant's functioning in the week prior to the evaluation.

Participants were rated on a 1-7 scale $(1=$ very minimal level of role functioning, $7=$ optimal level of role functioning) on the following areas: Work productivity, independent living, immediate social network, and extended social network. Interviewers achieved interrater reliability of .70 or higher (ICCs) for all subscales. A total RFS score was computed by summing across all subscales.

Information used for MCAS ratings was obtained via chart review and integration of all available study data (i.e. other assessments). From these sources, we were able to compute a social competence subscale (social acceptability, social interest, and social effectiveness items) and a modified adjustment to living subscale (independence of daily living and acceptance of illness items). Inter-rater reliability for both subscales was adequate (ICCs $\geq$. 70).

Social skill was evaluated via the Social Skills Performance Assessment (Patterson, Moscona, McKibbin, Davidson, \& Jeste, 2001). The SSPA consists of two, 3-minute role play conversations with a research confederate on pre-determined topics (e.g. meeting a new neighbor and requesting repairs from a landlord). SSPA performance was tape-recorded and scored by two coders, trained to reliability (ICCs $\geq .70$ ), and blind to treatment condition and assessment status (i.e. pre, post-test, 3-month follow-up). Although ratings were made on a variety of social skill indices (e.g. speech fluency and clarity), we focused on overall social appropriateness across the two scenes so as to reduce the number of variables for subsequent data analyses.

\subsection{Secondary Outcomes}

Interviewer-rated secondary outcomes measures included the three subscales (i.e., positive, negative, and general symptoms) and total score from the PANSS (Kay, Opler, \& 
Lindenmayer, 1988) and the Calgary Depression Scale for Schizophrenia (CDSS) (D. Addington, Addington, \& Maticka-Tyndale, 1993). Raters were blind to treatment condition and were trained using a series of "gold standard" videotapes (i.e., ICCs $\geq .80$ ).

Additional secondary outcomes included well-being, as measured by the environmental mastery (alpha =.89) and the purpose in life (alpha $=.83$ ) subscales from the Scales of Psychological Well Being (Ryff, 1989) (Note: These subscales were combined into a single scale as they were highly correlated $(r=.74, p<.01))$, social support, as measured by the Multidimensional Scale of Perceived Social Support (MSPSS) (alpha=.91) (Zimet, Powell, Farley, \& Werkman, 1990), alcohol and illicit drug use as assessed with the Alcohol Use Scale (AUS) and Drug Use Scale (DUS), respectively (Drake, Mueser, \& McHugo, 1996), and attitudes toward medications as measured by the Brief Evaluation of Medication Influence and Beliefs (BEMIB) (alpha=.72) (Dolder et al., 2004). The BEMIB has been shown to reliably and accurately identify individuals who are likely to be non-adherent with prescribed medication. Frequency and duration of readmission to the hospital was measured using participant self-report that was verified by chart review. Both the number and duration of hospitalizations were recorded.

Finally, we asked clients (who received GRIP only) and their study therapists to rate their progress in achieving goals on a " 1 " (no progress) to " 5 " (goal was achieved) likert scale, as well as to complete a brief questionnaire to provide feedback on GRIP following completion of the program (Waldheter et al., 2008).

\subsection{Procedures}

Clinical interviews evaluating psychiatric symptoms (PANSS), quality of life (QLS), substance use (AUS/DUS) and depression (CDSS) were conducted by assessors (a licensed clinical social worker and a licensed clinical psychologist) blind to treatment group. As a check on rater blindness, the clinical assessors were asked to guess participants' group assignment. The primary interviewer (social worker) correctly guessed group assignment for $54.3 \%$ of the participants, while the back-up interviewer (clinical psychologist) guessed correctly for $43.5 \%$ of the participants. These guesses appear to be within chance.

All recruitment procedures and self-report evaluations were conducted by two research assistants (i.e. a full-time staff person and a doctoral student in clinical psychology) who were not blind to treatment condition. These RAs were supervised weekly by the PI (DP) and managed the day-to-day running of the study, in addition to administering the self-report study measures (and rating client functioning, based on chart review).

The majority of participants in this study were referred by OASIS clinicians, with whom we met weekly during team meetings. Once potential participants were identified by their primary clinician and agreed to be contacted, they were screened over the phone in terms of general eligibility and interest in the study. Following the phone screen, potential participants were then formally interviewed to assess eligibility and obtain consent, and if they were deemed eligible, the participants were assessed on the outcome measures.

Participants who completed the screening and baseline interviews were randomly assigned to one of two conditions: 1) GRIP + TAU or 2) TAU alone. Randomization was stratified by gender to ensure equal numbers across conditions using a computer randomization generator. Participants were assessed at baseline, post-treatment, and at 3-month follow-up. Each participant who received TAU alone was yoked to a participant receiving GRIP, and post-test and follow-up assessments for each yoked pair were determined by the timeline of treatment completion for the GRIP participant. 


\subsection{Results}

\subsection{Data Analysis Overview}

Statistical analyses were conducted on the full, intent-to-treat, sample using mixed effects linear modeling to compare the two groups over time on the outcome variables listed above, with baseline performance as a covariate. The statistical analysis plans in the original grant application were to fit a random coefficient model; however, with only two post-baseline visits (GRIP versus TAU), we decided to treat the visit variable categorically and fit a more standard mixed model approach to repeated measures using an unstructured covariance structure among the repeated measures. This did not alter the results. The models included treatment, assessment point, treatment-by-assessment interaction, and baseline value of the response variable. Denominator degrees of freedom were calculated using the KenwardRoger method (Kenward \& Roger, 1997). Because we did not control for multiple comparisons, and we had multiple response variables, any treatment differences found were to be considered suggestive only. In addition, we computed within group effect sizes for all variables. These analyses should be considered exploratory given the small sample size associated with interventions at this stage of treatment development (Mueser \& Drake, 2005), which can result in large standard errors (Kraemer et al., 2006)

We conclude the data analysis section with a summary of participants' goal attainment, as well as feedback on GRIP, both of which are broken down by treatment completion status (i.e. treatment completers versus non-completers).

\subsection{Preliminary analyses}

During the active recruitment phase, 144 individuals were referred for the study (see Figure 1 for consort diagram). Fifty-six individuals were ineligible to participate, and 41 individuals refused to participate. Forty-seven individuals were screened, 46 of whom were then subsequently randomized (GRIP $=23$, TAU $=23$ ). In each treatment condition, 1 participant dropped out before the end of the study (i.e. did not complete post-test), and an additional participant dropped out before the 3-month follow-up assessment. Overall, $96 \%$ of the sample ( $\mathrm{N}=44)$ completed post-test, and $91 \%$ of all participants $(\mathrm{N}=42)$ completed the 3-month assessment, with no differential rates of attrition between GRIP and TAU.

In regard to tolerability, GRIP participants $(\mathrm{N}=23)$ attended a mean of 19 sessions ( $\mathrm{SD}=12.1$, range $=4-36)$, and $61 \%(\mathrm{~N}=14)$ of GRIP participants were considered to be treatment completers (i.e. attended at least 12 sessions, corresponding to Phase I of GRIP). Treatment completers attended a mean of 26 sessions $(\mathrm{SD}=9.7$, range $=12-36)$. Noncompleters attended a mean of 7 sessions $(\mathrm{SD}=2.5$, range $=4-11)$. Reasons for receiving less than 12 sessions included: 1) participant moved $(\mathrm{n}=3) ; 2)$ suicide $(\mathrm{n}=1) ; 3)$ inconsistent attendance $(\mathrm{n}=1) ; 4)$ participant did not want to participate in therapy anymore $(\mathrm{n}=3)$ and 5$)$ unknown $(\mathrm{n}=1)$. Treatment completers and non-completers did not differ on any baseline outcome variables.

Demographic characteristics of the study participants are summarized in Table 1. Sixty-one percent of participants in each group (GRIP + TAU and TAU) were male. Ethnic composition of the total sample was 63\% ( $\mathrm{N}=29)$ Caucasian, 28\% ( $\mathrm{N}=13)$ AfricanAmerican, and 9\% ( $\mathrm{N}=4)$ 'other'. Participants who identified ethnically as 'other' listed the following ethnicities: African ( $\mathrm{N}=1)$, Filipino $(\mathrm{N}=1)$, Cherokee Indian $(\mathrm{N}=1)$, and Hispanic $(\mathrm{N}=1)$. Hispanic enrollment included 3 males, one of whom identified as Caucasian, one as African-American, and one as 'other' (Hispanic). The average age of all participants was 22 years old ( $\mathrm{SD}=3.36$, range $=18-35$ ) (with GRIP participants being significantly older than TAU participants), 94\% were never married, and $93 \%$ had completed high school. The most common diagnosis was schizophrenia $(54 \%, \mathrm{~N}=25)$, followed by schizophreniform disorder 
(28\%, $\mathrm{N}=13)$, and schizoaffective disorder $(17 \%, \mathrm{~N}=8)$. The mean duration of time between onset of first sign or symptom of illness and date of first treatment (duration of untreated illness; DUI) was 23 months ( $\mathrm{SD}=26.52$ ), and the mean duration of time between onset of full syndrome and date of first treatment (duration of untreated psychosis; DUP) was 4 months ( $\mathrm{SD}=8.76)$.

Ninety-eight percent $(\mathrm{N}=45)$ of participants were prescribed at least one atypical antipsychotic medication over the course of the study, and $11 \%(\mathrm{~N}=5)$ of participants were prescribed both atypical and typical antipsychotic medications. Seventy-six percent $(\mathrm{N}=35)$ were prescribed an additional psychotropic medication in addition to an antipsychotic medication. One participant refused all medications for the duration of the study.

\subsection{Primary analyses}

The majority of the mixed model analyses were not statistically significant (in terms of group or group X time interactions). However, participants who received GRIP showed greater improvement in work functioning on the RFS at follow-up relative to TAU, while TAU participants showed greater improvement in perceived social support at follow-up relative to those who received GRIP. In addition, there was a trend $(\mathrm{p}<.10)$ in favor of GRIP participants showing a greater reduction in PANSS general symptoms at follow-up relative to those in TAU, and for TAU participants showing a greater reduction in depression (CDSS) through follow-up (and see section 5.4 for discussion of within-group change) (see Tables 2 and 3).

\subsection{Supplemental analyses}

In addition to the foregoing, we examined within-group changes and effect sizes. Table 2 summarizes the results for the functional outcomes. In general, effect sizes for GRIP both at post-test and at 3-month follow up are larger than those for TAU on the RFS subscales, QOL social subscale, and the MCAS social competence scale. Significant within group change was observed for GRIP on the RFS-extended social network and total, and the MCAS social competence scale (baseline to post-test; baseline to follow-up). Within group change on the RFS-immediate social network and QOL-social approached statistical significance (baseline to follow-up). Effect sizes were in the medium-large range.

The only functional variable to demonstrate a significant change for the TAU group was RFS-extended social network (baseline to follow-up). Within group change on the RFSTotal approached statistical significance (baseline to follow-up). These results suggest that GRIP might have a greater impact on functional outcomes than TAU, although data from two of the variables (i.e., RFS and MCAS) were obtained from non-blinded raters and we did not directly compare effect sizes across conditions.

Table 3 summarizes the results for the other study outcomes. Both groups demonstrated a significant reduction in depression from baseline through follow-up on the CDSS. The TAU group reported improved social support through follow-up and better attitudes toward medications through post-treatment. The GRIP group had higher effect sizes through followup on the PANSS than the TAU group, but within group change was not statistically significant. Finally, the GRIP group demonstrated an unexpected worsening of alcohol use from baseline to follow-up.

Table 4 summarizes the results for the hospitalization data. The TAU group had twice as many hospitalizations and was hospitalized for more days than the GRIP group. It should be noted, however, that the latter finding was greatly influenced by one TAU participant who had been hospitalized for 100 days. 


\subsection{Goal attainment}

Of the 22 participants in the GRIP condition who completed post-test, clients set an average of 3.18 goals (SD 1.37, range 1-6). Fifty-eight percent of all personal goals were rated by participants at post-test as "very close to being achieved" or "achieved." Further analyses revealed a difference between GRIP completers and non-completers. While treatment completers collectively rated $65 \%$ of their personal goals at post-test as "very close to being achieved" or "achieved," only $43 \%$ of the treatment non-completers' goals received those same ratings.

Therapists rated $51 \%$ of client goals as being "very close to being achieved" or "achieved" by post-test. Similar to the client ratings made by treatment completers, therapists rated $65 \%$ of treatment completers' goals as "very close to being achieved" or "achieved." However therapists assessed that only $19 \%$ of non-completers' goals met this same standard.

\subsection{Qualitative feedback}

Table 5 summarizes the qualitative ratings from GRIP participants. Overall, all participants gave GRIP positive ratings, especially those who completed a minimum of 12 sessions.

\subsection{Discussion}

The purpose of this study was to examine the feasibility and tolerability of GRIP, as well as to obtain specific treatment parameters (e.g. effect sizes) to inform the implementation of a larger, more definitive RCT. We hypothesized that GRIP would be well-tolerated and received, and that it would be associated with a greater reduction in symptoms, improvement in functioning, and lower hospitalization rates than TAU alone.

Overall, GRIP appears to be a feasible and well-tolerated intervention. Drop-out rates were low and attendance rates, particularly for treatment "completers" were high. In fact, our drop-out rates compare favorably to other individual therapy studies for first episode psychosis (J Edwards et al., 2006; Gleeson et al., 2009; H. Jackson et al., 2005; H. J. Jackson et al., 2008; Kavanagh et al., 2004; Power et al., 2003; Nick Tarrier et al., 2004). In addition, feedback from participants was consistently positive (based on their questionnaire ratings), suggesting that GRIP was meeting their treatment needs. Thus, GRIP appears to be an acceptable intervention to clients with first episode psychosis.

In regard to the primary and secondary outcomes, the lack of significant effects observed in the mixed models (with the exception of the work productivity scale on the RFS) might suggest that GRIP did not add clinical value beyond treatment as usual. However, these findings may be due to lack of statistical power, since by and large, the results were in the hypothesized direction and the effect sizes were not insubstantial. And, this was a pilot study and was not statistically powered to definitively test these hypotheses. Most of the participants in the RCT (44/46) were clients of the OASIS program, which offers comprehensive services for clients with first episode psychosis. Thus, it is possible that the therapeutic effects of a specialty first episode program account for most of the variance in client improvement, thus setting a high bar for individual therapy to make an impact.

Our secondary analyses examined effect sizes and within-group changes. These analyses suggest that GRIP might have an impact on functional outcomes, as participants who received GRIP showed significant within group change through 3-month follow-up on role functioning (as measured by the RFS) and social competence (as measured by the MCAS), and trend level improvement in quality of life. These effect sizes were, for the most part, consistently larger than those observed for participants who received TAU, and were in the small to medium range. In addition, the GRIP group had higher effect sizes through follow- 
up on the PANSS than the TAU group, but the within group change was not statistically significant. Although the GRIP group showed an increase in alcohol use from baseline to follow-up, post-hoc analyses revealed that only treatment non-completers had a significant increase in alcohol use. This suggests that alcohol use may have a relationship with treatment engagement and retention. Finally, GRIP participants were hospitalized less frequently than those who received TAU, although these differences were not statistically significant. Thus, the pattern of findings seems to favor GRIP over TAU in improving the functioning of clients with first episode psychosis, and indicate that this treatment may be worth further investigation

In general, GRIP did not have an effect on social support, beliefs about medications, and psychological well-being. Interestingly, individuals who received TAU alone did show unexpected improvement in these areas, particularly social support and beliefs about medications, as well as a reduction in depression. These findings underscore the potential effectiveness of multi-element treatment programs for first episode psychosis, but they don't explain the lack of an effect for the group that received GRIP. It is possible that a focused discussion on medication adherence (as done in Phase I of GRIP), may not be effective approach for individuals with first episode psychosis. In regard to social support, the TAU group was (non-significantly) lower than the GRIP group at baseline, with only 1.46 points separating the two groups at 3-month follow-up, so regression to the mean is also a possibility. The important point, however, is that GRIP did not impact social support, beliefs about medications, and psychological well-being suggesting that changes need to be made to the intervention in order to improve its effectiveness in these domains.

The findings also emphasize the importance of treatment engagement for clients with first episode psychosis. Treatment "completers" tended to be more successful at achieving their goals relative to "non-completers." This was true regardless of whether goal attainment was rated by clients themselves or their clinicians. This is not a trivial issue, as goal attainment is linked to mental health (King, 2001). Thus, strategies to increase treatment engagement are potentially critical to improving client's quality of life and well-being.

This study has a number of strengths and weaknesses which should be acknowledged. Strengths include a well-developed intervention, clearly differentiated treatment conditions, utilization of psychometrically sound measures, low drop-out rates, and raters blind to treatment assignment for the clinical (PANSS, CDSS) and quality of life assessments. Weaknesses include the small sample size of the pilot RCT, the lack of rater blindness for two of the functional outcome measures (RFS and MCAS), the short follow-up period, and measures which may not have been sensitive to change, were not directly linked with the intervention (e.g. mood; social support), and did not adequately capture recovery from the client's perspective.

In closing, the results of this study indicate that GRIP is a feasible intervention for first episode psychosis and is well-tolerated by clients. Both GRIP and TAU, which was provided at a comprehensive specialty first episode program, resulted in improvements across both functional and clinical domains, although evaluation of effect sizes suggest an advantage for GRIP in improving functional outcomes (which again, need to be interpreted cautiously in a preliminary study, (Kraemer et al., 2006). Thus, the results of this small scale RCT suggest that GRIP has promise and that a larger, more definitive clinical trial may be warranted.

\section{Acknowledgments}

The authors would like to thank the clients, clinicians, and researchers who have contributed to the development and evaluation of GRIP. We also acknowledge the assistance of Abby Scheer for providing data management 
services, Brent Moos, LCSW and Lyse Debourguignon, LCSW, for serving as GRIP therapists, Jennifer Nieri, LCSW, Piper Meyer, Ph.D, and Leanne Whaley Owens, B.A. for conducting study assessments, Bebe Smith, LCSW, Karen Graham, M.D. and Lisa Cooper, for administrative and clinical support at OASIS, and the Duke Endowment and KB Reynolds Foundations, for providing support for the development of OASIS. Support for the development and evaluation of GRIP has been provided by NIMH grant R34 1-MH071252-01A1 awarded to DLP.

\section{References}

Addington D, Addington J, Maticka-Tyndale E. Assessing depression in schizophrenia: The Calgary Depression Scale. British Journal of Psychiatry 1993;163 Suppl 22:39-44.

Addington J, Gleeson J. Implementing cognitive-behavioural therapy for first-episode psychosis. British Journal of Psychiatry 2005;187 suppl. 48:s72-s76.

Birchwood M, Todd P, Jackson C. Early intervention in psychosis: The critical period hypothesis. British Journal of Psychiatry 1998;172 Suppl. 33:53-59. [PubMed: 9534833]

Dickerson FB, Origoni AE, Pater A, Friedman BK, Kordonski WM. An expanded version of the Multnomah Community Ability Scale: Anchors and interview probes for the assessment of adults with serious mental illness. Community Mental Health Journal 2003;39(2):131-137. [PubMed: 12723847]

Dolder CR, Lacro JP, Warren KA, Golshan S, Perkins DO, Jeste DV. Brief Evaluation of Medication Influences and Beliefs: Development and Testing of a Brief Scale for Medication Adherence. Journal of Clinical Psychopharmacology 2004;24(4):404-409. [PubMed: 15232332]

Drake, RE.; Mueser, KT.; McHugo, GJ. Clinician rating scales: Alcohol Use Scale (AUS), Drug Use Scale (DUS), and Substance Abuse Treatment Scale (SATS). In: Sederer, LI.; Dickey, B., editors. Outcomes assessment in clinical practice. Baltimore: Williams \& Wilkins; 1996. p. 113-116.

Edwards J, Elkins K, Hinton M, Harrigan S, Donovan K, Athanasopoulos O, et al. Randomized controlled trial of a cannabis-focused intervention for young people with first-episode psychosis. Acta Psychiatric Scandinavica 2006;114(2)

Edwards, J.; Hinton, M.; Elkins, K.; Anthanasopoulos, O. Cannabis and first-episode psychosis: The CAP project. In: Graham, H.; Mueser, KT.; Birchwood, M.; Copello, A., editors. Substance misuse in psychosis: Approaches to treatment and service delivery. Chichester: John Wiley \& Sons; 2003. p. 283-304.

Fowler D, Hodgekins J, Painter M, Reilly T, Crane C, Macmillan I, et al. Cognitive behaviour therapy for improving social recovery in psychosis: a report from the ISREP MRC Trial Platform study (Improving Social Recovery in Early Psychosis). Psychological Medicine 2009;39(10):1627-1636. [PubMed: 19335932]

Gleeson JFM, Cotton SM, Alvarez-Jimenez M, Wade D, Gee D, Crisp K, et al. A randomized controlled trial of relapse prevention therapy for first-episode psychosis patients. Journal of Clinical Psychiatry 2009;70(4):477-486. [PubMed: 19323964]

Goodman SH, Sewell DR, Cooley EL, Leavitt N. Assessing levels of adaptive functioning: The Role Functioning Scale. Community Mental Health Journal 1993;29(2):119-131. [PubMed: 8500285]

Haddock G, Lewis S. Psychological Interventions in Early Psychosis. Schizophrenia Bulletin 2005;31(3):697-704. [PubMed: 16006594]

Harvey P-O, Lepage M, Malla A. Benefits of enriched intervention compared with standard care for patients with recent-onset psychosis: a metaanalytic approach. Canadian Journal of Psychiatry 2007;52:464-472.

Heinrichs DW, Hanlon TE, Carpenter WT. The Quality of Life Scale: An instrument for rating the schizophrenic deficit syndrome. Schizophrenia Bulletin 1984;10(3):388-398. [PubMed: 6474101]

Jackson C, Trower P, Reid I, Smith J, Hall M, Townend M, et al. Improving psychological adjustment following a first episode of psychosis: a randomized controlled trial of cognitive therapy to reduce post psychotic trauma symptoms. Behaviour Research and Therapy 2009;47:454-462. [PubMed: 19361782]

Jackson H, McGorry P, Edwards J, Hulbert C, Henry L, Harrigan S, et al. A controlled trial of cognitively oriented psychotherapy for early psychosis (COPE) with four-year hollow-up readmission data. Psychological Medicine 2005;35:1-12. 
Jackson HJ, McGorry PD, Killackey E, Bendall S, Allott K, Dudgeon P, et al. Acute phase and 1-year follow-up results of a randomized controlled trial of CBT versus befriending for first-episode psychosis: The ACE project. Psychological Medicine 2008;38(5):725-735. [PubMed: 18005494]

Kavanagh DJ, Young R, White A, Saunders JB, Wallis J, Shockley N, et al. A brief motivational intervention for substance misuse in recent-onset psychosis. Drug and Alcohol Review 2004;23:151-155. [PubMed: 15370020]

Kay SR, Opler LA, Lindenmayer J-P. Reliability and validity of the Positive and Negative Syndrome Scale for schizophrenics. Psychiatry Research 1988;23(1):99-110. [PubMed: 3363019]

Kenward MG, Roger JH. Small Sample Inference for Fixed Effects from Restricted Maximum Likelihood. Biometrics 1997;53(3):983-997. [PubMed: 9333350]

King LA. The health benefits of writing about life goals. Personality and Social Psychology Bulletin 2001;27(7):798-807.

Kraemer HC, Mintz J, Noda A, Tinklenberg J, Yesavage JA. Caution Regarding the Use of Pilot Studies to Guide Power Calculations for Study Proposals. Archives of General Psychiatry 2006;63(5):484-489. [PubMed: 16651505]

Lecomte T, Leclerc C, Corbière M, Wykes T, Wallace CJ, Spidel A. Group cognitive behavior therapy or social skills training for individuals with a recent onset of psychosis? Results of a randomized controlled trial. Journal of Nervous and Mental Disease 2008;196(12):866-875. [PubMed: 19077853]

Lewis S, Tarrier N, Haddock G, Bentall R, Kinderman P, Kingdon D, et al. Randomised controlled trial of cognitive-behavioural therapy in early schizophrenia: Acute-phase outcomes. British Journal of Psychiatry 2002;181 Suppl. 43:s91-s97.

Malla AK, Norman RM, Joober R. First-episode psychosis, early intervention, and outcome: What have we learned? Canadian Journal of Psychiatry 2005;50(14):881-891.

Mueser, KT.; Drake, RE. How Does a Practice Become Evidence-Based?. In: Drake, RE.; Merrens, MR.; Lynde, DW., editors. Evidence-based mental health practice: A textbook. New York, NY US: W W Norton \& Co; 2005. p. 217-241.

Patterson TL, Moscona S, McKibbin CL, Davidson K, Jeste DV. Social skills performance assessment among older patients with schizophrenia. Schizophrenia Research 2001;48(2-3):351-360. [PubMed: 11295387]

Penn DL, Waldheter EJ, Perkins DO, Mueser KT, Lieberman JA. Psychosocial treatment for firstepisode psychosis: A research update. American Journal of Psychiatry 2005;162:2220-2232. [PubMed: 16330584]

Power P, Bell RJ, Mills R, Herrman-Doig T, Davern M, Henry L, et al. Suicide prevention in first episode psychosis: The development of a randomised controlled trial of cognitive therapy for acutely suicidal patients with early psychosis. Australian and New Zealand Journal of Psychiatry 2003;37:414-420. [PubMed: 12873325]

Ryff CD. Happiness is everything, or is it? Explorations on the meaning of psychological well-being. Journal of Personality and Social Psychology 1989;57(6):1069-1081.

Saksa JR, Cohen SJ, Srihari VH, Woods SW. Cognitive behavior therapy for early psychosis: A comprehensive review of individual vs. group treatment studies. International Journal of Group Psychotherapy 2009;59(3):357-383. [PubMed: 19548785]

Tarrier N, Haddock G, Lewis S, Drake R, Gregg L. Suicide behaviour over 18 months in recent onset schizophrenic patients: The effects of CBT. Schizophrenia Research 2006;83:15-27. [PubMed: 16460916]

Tarrier N, Lewis S, Haddock G, Bentall R, Drake R, Kinderman P, et al. Cognitive-behavioural therapy in first-episode and early schizophrenia: 18-month follow-up of a randomised controlled trial. British Journal of Psychiatry 2004;184(3):231-239. [PubMed: 14990521]

Tauber R, Wallace CJ, Lecomte T. Enlisting indigenous community supporters in skills training programs for persons with severe mental illness. Psychiatric Services 2000;51(11):1428-1432. [PubMed: 11058191]

The Psychological Corporation. Manual for the Wechsler Abbreviated Scale of Intelligence. San Antonio, TX: The Psychological Corporation; 1999. 
Uzenoff SR, Perkins DO, Hamer RM, Wiesen CA, Penn DL. A preliminary trial of adherence-copingeducation (ACE) therapy for early psychosis. Journal of Nervous and Mental Disease 2008;196(7): 572-575. correction, 196(510), 780. [PubMed: 18626299]

Waldheter EJ, Penn DL, Perkins DO, Mueser KT, Owens LW, Cook E. The Graduated Recovery Intervention Program for first episode psychosis: treatment development and preliminary data. Community Mental Health Journal. 2008 May 31 Epub ahead of print.

Zimet GD, Powell SS, Farley GK, Werkman S. Psychometric characteristics of the Multidimensional Scale of Perceived Social Support. Journal of Personality Assessment 1990;55(3-4):610-617. [PubMed: 2280326] 


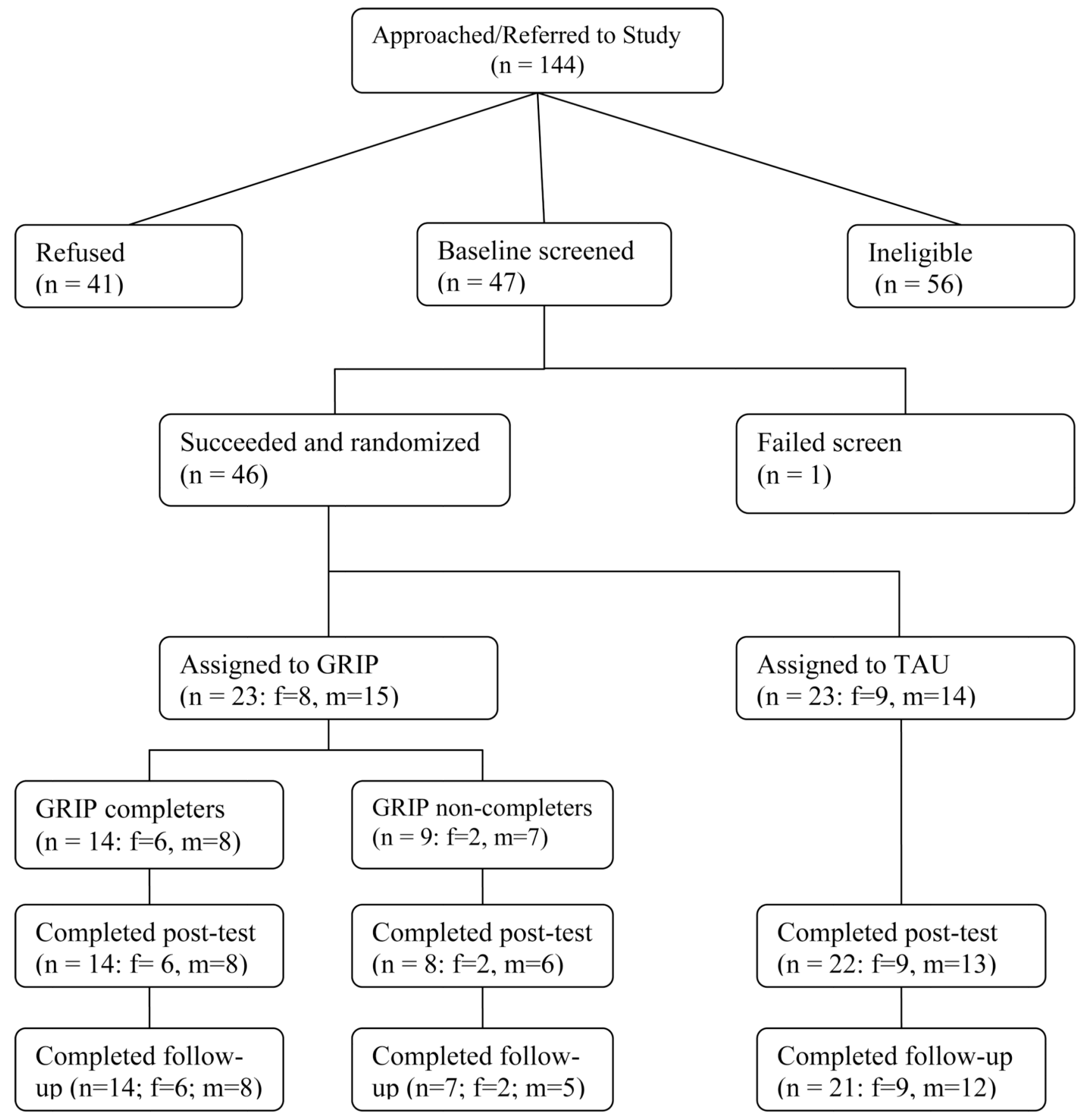

Figure 1.

GRIP RCT Consort Diagram 


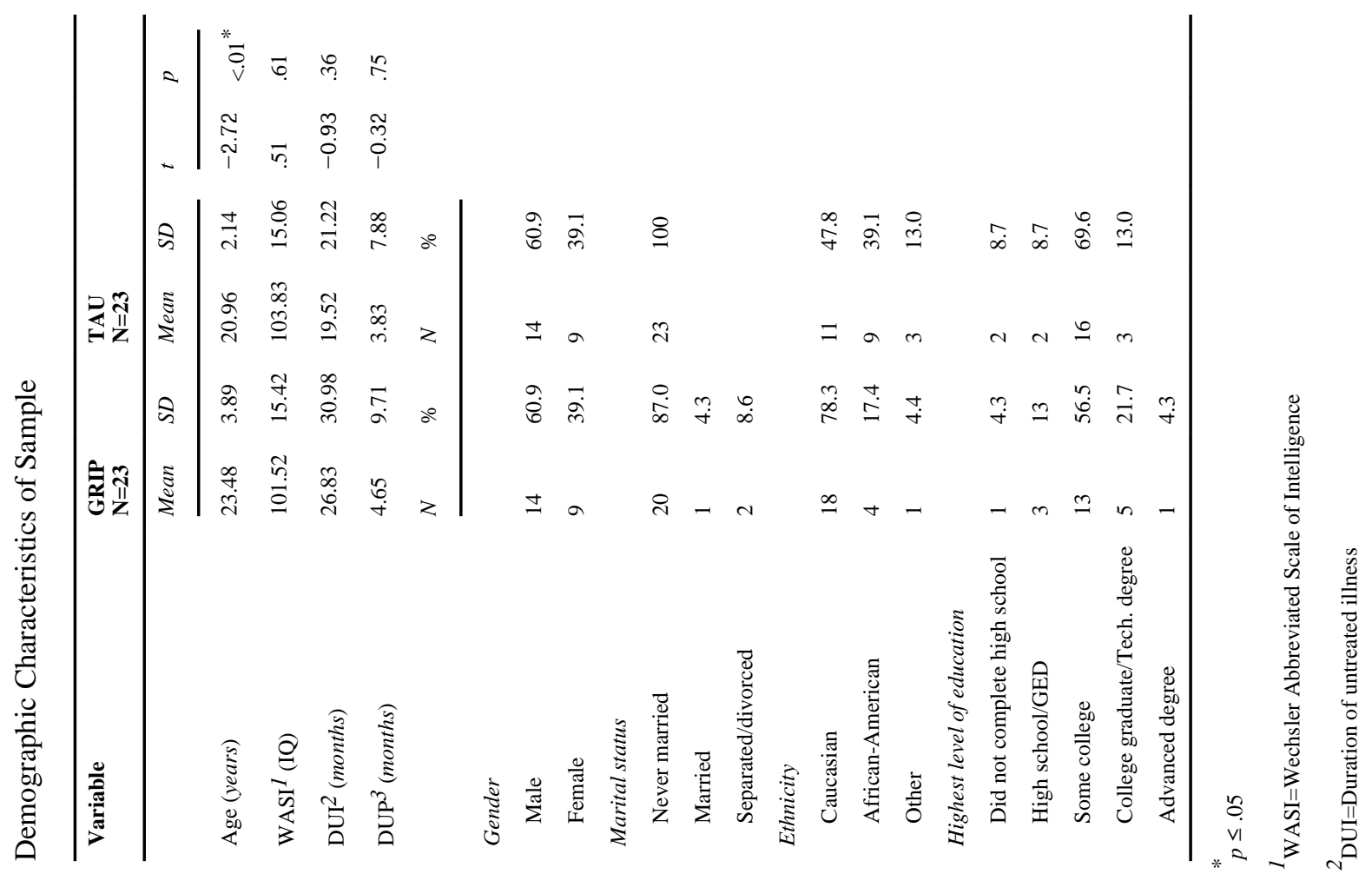




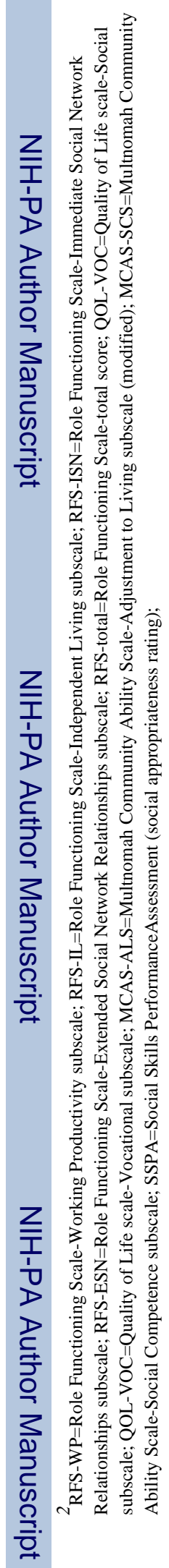

Schizophr Res. Author manuscript; available in PMC 2012 February 1. 


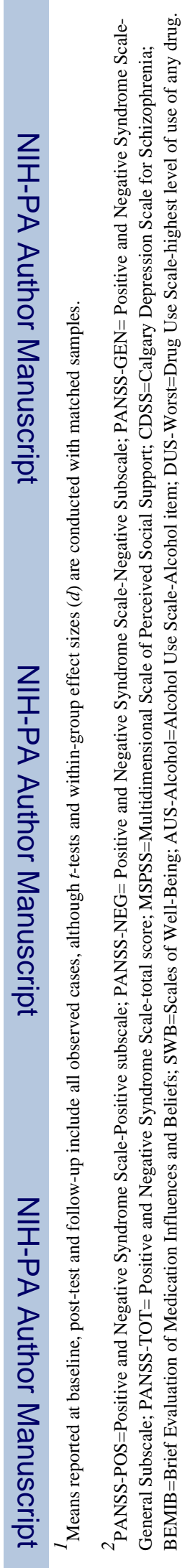




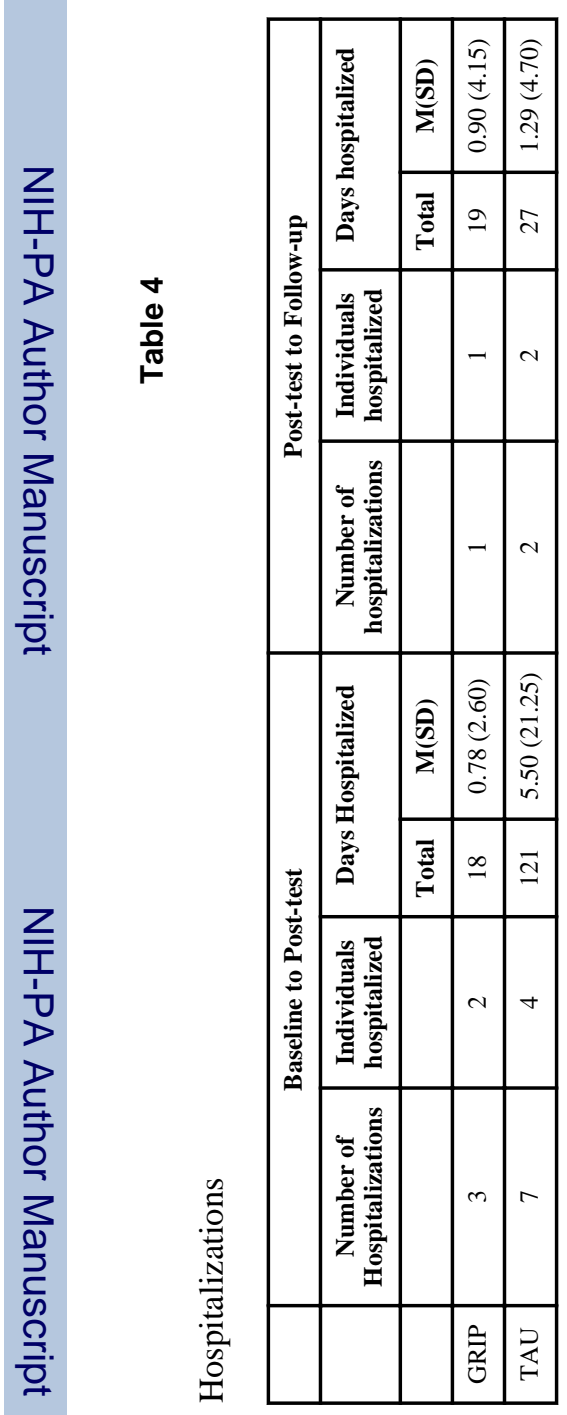

Schizophr Res. Author manuscript; available in PMC 2012 February 1. 


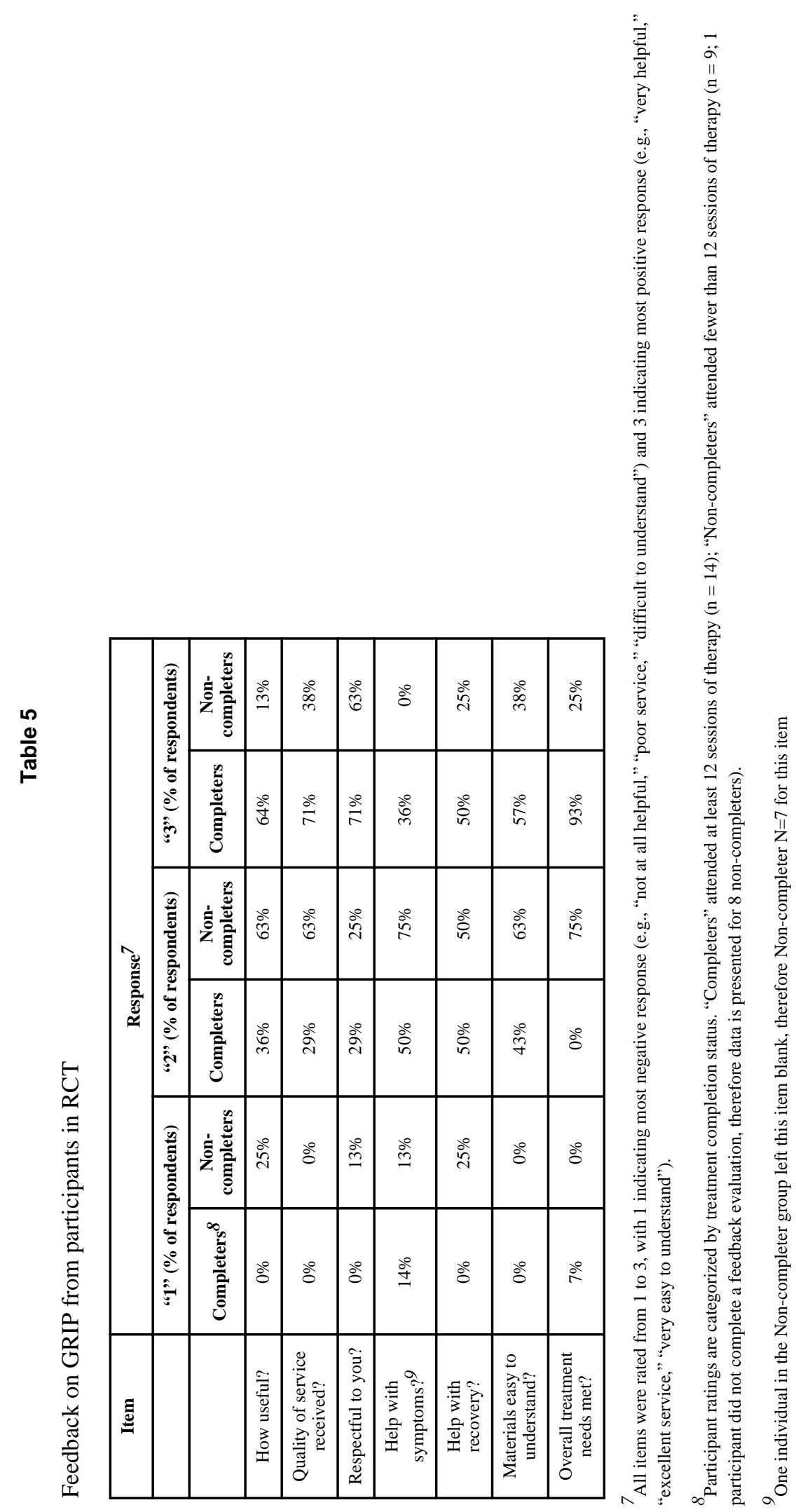

